\title{
Erratum
}

Stomatologie $2015 \cdot 112: 180$

DOI 10.1007/s00715-015-0056-3

Online publiziert: 24. Juni 2015

(c) Springer-Verlag Wien 2015
Simone Heuberer ${ }^{1} \cdot$ G. Watzak ${ }^{1}$ W. Zechner ${ }^{1} \cdot$ K. Zauza ${ }^{2} \cdot$ C. Ulm ${ }^{1} \cdot$ J. Freudenthaler

${ }^{1}$ Fachbereich Orale Chirurgie, Spezialambulanz „Aplasie", Universitätszahnklinik, Wien, Österreich

${ }^{2}$ Fachbereich Unit Zahnärztliche Ausbildung, Universitätszahnklinik, Wien, Österreich

${ }^{3}$ Fachbereich Kieferorthopädie, Universitätszahnklinik, Wien, Österreich

\section{Erratum zu: Zahnaplasien - ein Leitfaden}

\section{Erratum zu:}

Stomatologie (2015)

DOI 10.1007/s00715-015-0042-9

In der Online-Version dieses Beitrags wurde der Name von G. Watzak leider zunächst falsch veröffentlicht. Wir bitten die nun korrekten Angaben zu berücksichtigen und den Fehler zu entschuldigen.

Die Redaktion

\section{Korrespondenzadresse}

\section{Dr. S. Heuberer}

Fachbereich Orale Chirurgie

Spezialambulanz „Aplasie"

Universitätszahnklinik,

Sensengasse 2, 1090 Wien

simone.heuberer@meduniwien.ac.at 